Journal of Computer Science 6 (8): 934-939, 2010

ISSN 1549-3636

(C) 2010 Science Publications

\title{
Response of SAI Afferents May Play a Role in the Perception of Velvet Hand Illusion
}

\author{
${ }^{1}$ Abdullah Chami, ${ }^{1}$ Masahiro Ohka, ${ }^{1}$ Yuji Kawabe and ${ }^{2}$ Hanafiah Bin Yussof \\ ${ }^{1}$ Department of Complex Systems Science, Graduate School of Information Science, \\ Nagoya University, Furo-Cho, Chikusa-Ku, Nagoya 464-8601, Japan \\ ${ }^{2}$ Faculty of Mechanical Engineering, University Technology MARA, Malaysia
}

\begin{abstract}
Problem statement: We revealed the reason behind a haptic illusion called the Velvet Hand Illusion (VHI) by FEM analysis. In VHI, a person rubs his/her hands together on both sides of wires strung through a frame and produces the sensation of rubbing a very smooth and soft surface like velvet. We focus on VHI to determine the specifications of an actuator for a tactile display enhanced by VHI. Approach: A simulated fingertip was modeled under the same contact condition that an actual finger was believed to undergo when under VHI. We collected the simulated responses of a number of SAI afferents, which were responsible for detecting the edges, to relate them to the mechanism of the illusion. Results: Even with a simple model that treats only one finger, we noticed a considerable difference between the responses of a number of SAI receptors compared with that of a fingertip touching wires without the effect of the opposing finger. The SAI responses for the VHI case are less (some receptors' response is up to 50\% less) than those for a finger touching the wires, which corresponds to a decrease of the perception of the wires. Conclusion/Recommendations: SAI might play a role in the mechanism of the VHI illusion. This study could benefit the development of a haptic display that utilizes the illusion and makes a person experience the same feeling using the proposed display.
\end{abstract}

Key words: FEM analysis, haptic illusion, fingertip, merkel disk receptor

\section{INTRODUCTION}

Understanding the reasons behind sensory illusions can provide valuable information about human perception mechanisms. This information can be used in the development of both bio-inspired sensors (e.g., tactile sensors) and human-machine interface devices (e.g., haptic displays).

Because of its excellent dexterity and its ability to detect such different tactile cues as edges, textures and hardness, the human tactile system has tempted researchers to implement some of its characteristics in the design of tactile sensors (Zhang et al., 2006; Herrera, 2007).

Also interest in virtual environments, specifically in haptic display technologies is increasing because being able to touch, feel and manipulate objects in addition to seeing and hearing them is essential for realizing the full promise of virtual environments (Bresciani et al., 2008). However, to date, since haptic devices can't generate a copy of "the actual stimulation" to give perception of the real world, the investigation of other approaches remains more appealing.

Considering the characteristics of human tactile perception, haptic displays do not necessarily have to generate a sensory flow that strictly corresponds to the actual stimulation that leads to this percept in the "real" world, but "only" a sensory flow that elicits this percept (Bresciani et al., 2008).

In other words, if a haptic illusion is utilized in the design of a haptic display, it does not need to generate "stimulation A" that actually causes the "complex perception", but only a simpler "stimulation B" that is only necessary to trick or "illude" the person into sensing the same complex perception that "stimulation A" elicits.

Until recently, however, haptic illusions failed to get the attention compared to other sensory illusions: namely visual illusions. Such visual illusions as the well-known barber pole illusion, the Ouchi illusion and the MullerLyer illusion have been extensively studied. Recently a set of psychophysical experiments studied the "haptic versions" of these illusions (Scilingo et al., 2008).

Corresponding Author: Abdullah Chami, Department of Complex Systems Science, Graduate School of Information Science, Nagoya University, Furo-Cho, Chikusa-Ku, Nagoya 464-8601, Japan

Tel: +81-52-789-4861 Fax: +81-52-789-4800 


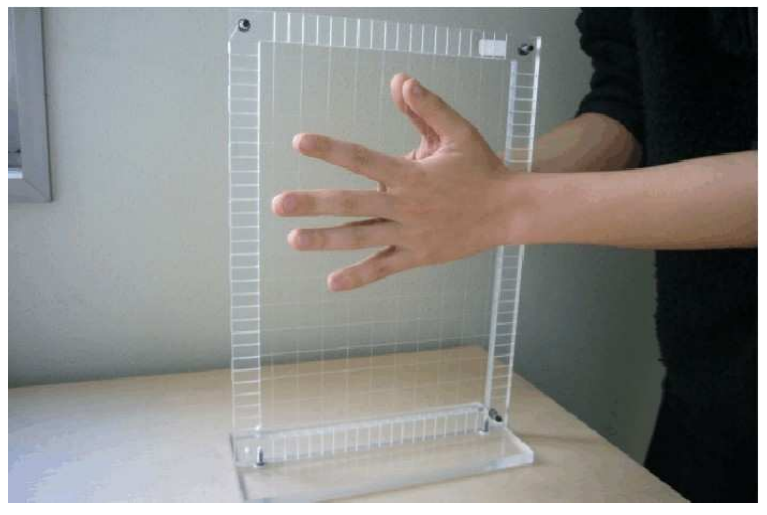

Fig. 1: Wire mesh generating velvet hand illusion

For instance, in the visual Muller-Lyer illusion, a person perceives images that differ from objective reality. The tactile Muller-Lyer is caused by relief-like figure. When judging the length of two equal convex lines by touching them, a person typically claims that the line with inward-pointing arrows is longer. Besides the Muller-Lyer illusion in tactile sensation, there are several tactile illusions such as the Velvet Hand Illusion (VHI) (Mochiyama et al., 2005) and the fishbone and comb illusions (Hayward, 2008).

Our interest in the velvet hand illusion is mainly derived from the perception it elicits, touching a given material (velvet), so studying its mechanism and the reason behind it might enable us to utilize it in the design of a haptic display. VHI is caused by such a wire mesh Fig. 1. A person rubs his/her hands together on both sides of wires strung through a frame, producing the sensation of rubbing a very smooth and soft velvetlike texture.

In this study we predict the responses of a number of SAI afferents (Merkel disks) in a fingertip subjected to VHI using FEM analysis. The modeled response can then be used in the design of a haptic display to generate a stimulation that delivers the same response (i.e., the same perception) to the person touching it.

\section{MATERIALS AND METHODS}

\section{Modeling procedure:}

Background: When we contact an object with our skin, it deforms according to the shape of that object and due to the layered structure of our skin, the stress-strain state generated inside the skin is believed to be optimized to deliver mechanical stimulation to the mechanoreceptors, which are strategically located at the boundaries of those layers (Maeno et al., 1998).
The mechanoreceptors transform the mechanical stimulation into a series of neural pulses "action potential" that carries the stimulation to the brain. One type of these mechanoreceptors is called "Slowly Adapting type I" (SAI), which is known to encode curvatures and edges (Lesniak and Gerling, 2009).

Researchers proposed both continuum models (Srinivasan, 1989; Sripati et al., 2006) and finite element models (Maeno et al., 1998; Gerling and Thomas, 2005; 2008; Wu et al., 2006) to determine the stress-strain state inside the skin and tried to correlate a mechanical value (either stress or strain based) generated inside the fingertip at the location of the SAI afferents with the action potential generated by them that was measured in monkeys (Phillips and Johnson, 1981) or in man (Johansson and Flanagan, 2009).

In some reports (Dandekar et al., 2003; Lesniak and Gerling, 2009; Sripati et al., 2006) good fitness between the rate of spikes fired by an SAI afferent and the Strain Energy Density (SED) at the location of these afferents was achieved; this result has become the typical value to account for SAI response.

Lesniak and Gerling (2009) combined a FEM model of the fingertip and a neuron model to study the response of a single SAI receptor and compared it with the results of psychophysical data obtained by Phillips and Johnson (1981).

The FEM model: Although recently more complex models that account for the nonlinear elasticity and the time-dependent mechanical properties of the skin tissue were proposed (Wu et al., 2006) and used to study the fingertip under vibration or large displacement, linear models are sufficient for simulating the fingertip under contact in low speed (low frequency) and for small displacements around $1 \mathrm{~mm}$ (Lesniak and Gerling, 2009).

In this study we use a two-dimensional plane strain linear layered FEM model that represents a cross section of an average human index fingertip. Our model resembles the one used in Gerling and Thomas (2008) and Lesniak and Gerling (2009) without simulating the collagen fibers.

The fingertip is considered elliptical with a long axis of $17.44 \mathrm{~mm}$ and a short axis of $13.6 \mathrm{~mm}$ (Maeno et al., 1998). In addition to the bone and nail, it is assumed to consist of three soft tissue layers, starting from the outer one: epidermis, dermis and the subcutaneous tissue.

Figure 2 shows the thickness used for every layer in addition to the boundary between the epidermis and dermis layers. The boundary between the two layers has sinusoid shapes called the intermediate ridges. 


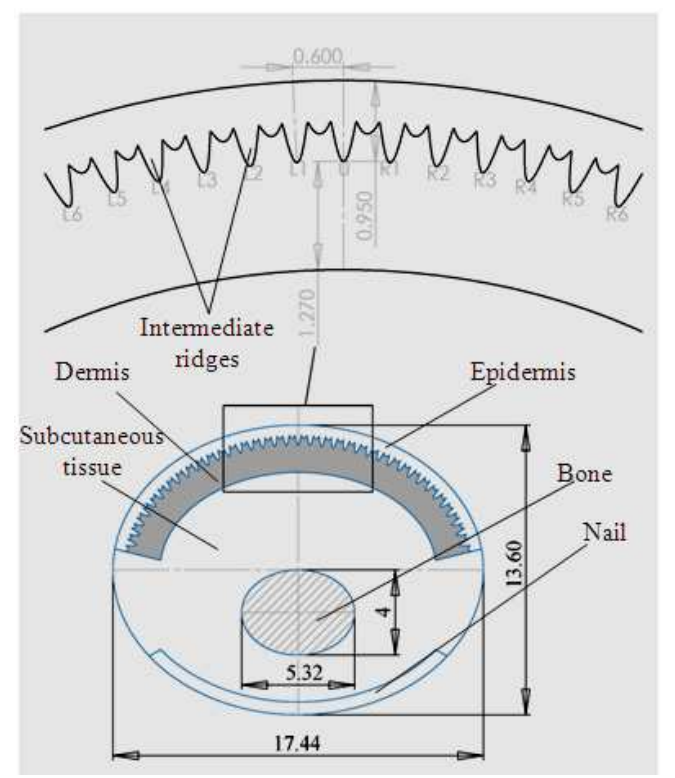

Fig. 2: Geometry of fingertip model (dimensions are in $\mathrm{mm}$ )

The elements located at their tips are considered the location where SAI receptors are located and the SEDs of these elements are considered the responses of the afferents discussed above.

We numbered the center one Rec. 0 (for receptor number 0), Rec. Li (for receptor on left side letter i) and Rec. Ri (for receptor on right side letter $\mathrm{i}$ ), where $\mathrm{i}=1 \sim 8$ with numbering starting from the one next to the center receptor going in both directions.

The soft tissues layers are assumed to be linearly elastic with a young modulus of $0.136 \mathrm{MPa}$ for the epidermis, $0.08 \mathrm{MPa}$ for the dermis and $0.034 \mathrm{MPa}$ for the subcutaneous tissue. Poisson's ratio is assumed as 0.48 for all layers (Maeno et al., 1998). Both the bone and the nail are assumed to be rigid. The model contains approximately 17,300 elements and 46,800 nodes. The simulation was performed by ANSYS Academic Release 12.0.1.

Simulating VHI: The following are the minimum conditions for VHI to be felt: (1) the use of both hands, (2) the presence of at least two wires either parallel or perpendicular to the fingers and (3) rubbing the hands against the wires (Mochiyama et al., 2005).

Although the reasons behind this illusion remain unclear, we believe that the repeated contact between one finger and the other and then with the wire triggers Fast Adapting type I (FAI) Afferents and that the shape and the dimensions of the wires (small and edge-like) also stimulate SAI. The combination of these two responses could function as a perception of VHI.

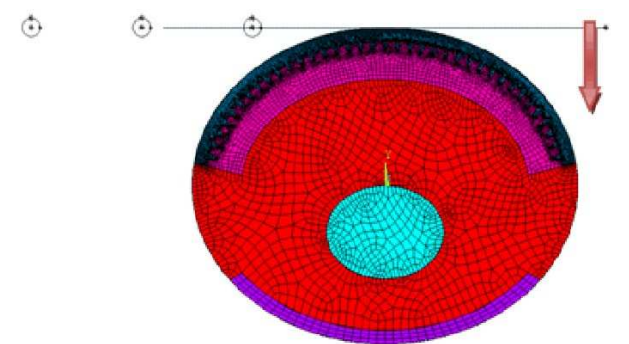

(a)

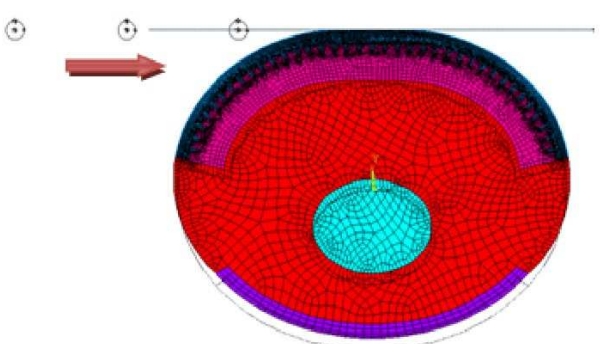

(b)

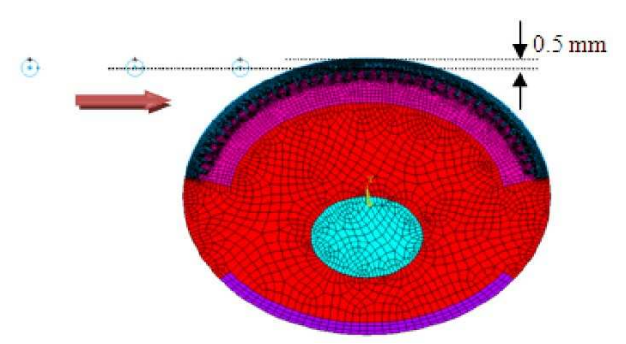

(c)

Fig. 3: FEM model: (a) Start of VHI case, (b) VHI case after compression and (c) start of one finger case

Here we tackle the SAI response alone by simulating a simple version of VHI on just two opposing fingers instead of the whole hand (VHI case) and compare with a finger touching the wires without the existence of a second finger (one-finger case).

VHI case simulation: This simulation starts by pressing the finger with a rigid plane with a displacement of $0.5 \mathrm{~mm}$ to account for the opposing finger effect (Fig. 3a and b) and then the wires ( $\mathrm{D}=0.8 \mathrm{~mm}$ and simulated as rigid bodies), which have their centers aligned with the fingertip surface have their centers aligned with the fingertip surface, move horizontally at $21 \mathrm{~mm} \mathrm{sec}^{-1}$ and deform the already pressed finger. The friction between the wires and the fingertip surface is assumed to be 0.3 . We use 3 wires with a pitch of $5 \mathrm{~mm}$. The boundary condition constrains the two nodes near the middle of the nail in both the $\mathrm{X}$ and $\mathrm{Y}$ directions. 
One-finger case simulation: For this case there is no compression because we assume no opposing finger. The three wires have their centers at a distance of 0.5 $\mathrm{mm}$ vertically from the top point of the surface of the fingertip (Fig. 3c) to insure that the difference between the two cases is only due to the compression by the rigid plane (the opposing finger effect) and not because of a different compression induced by the wires. The other parameters are the same as the VHI case.

\section{RESULTS}

As explained above and shown in Fig. 3 the wires contact the fingertip on the left side, deform it and leave the contact area from the right side, which means that the receptors on the fingertip's left side are stimulated first. The SEDs collected from the elements at the locations of the SAI receptors are shown in Fig. 4 and 5. On the right of Fig. 4 and 5 the SEDs for the VHI case is shown and the SEDs for the one-finger case is shown on the left.

Figure 4 illustrates the SEDs of the receptors on the left side of the fingertip in addition to the center receptor (a-Recs. L5 L8, b-Recs. L1 L4 and Rec. 0) and Fig. 5

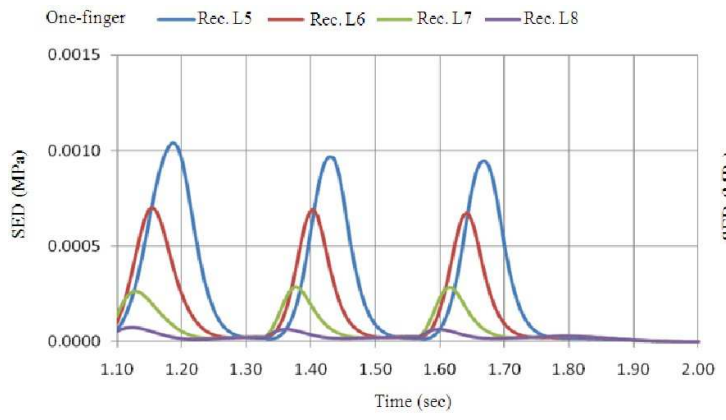

(a) Group 1
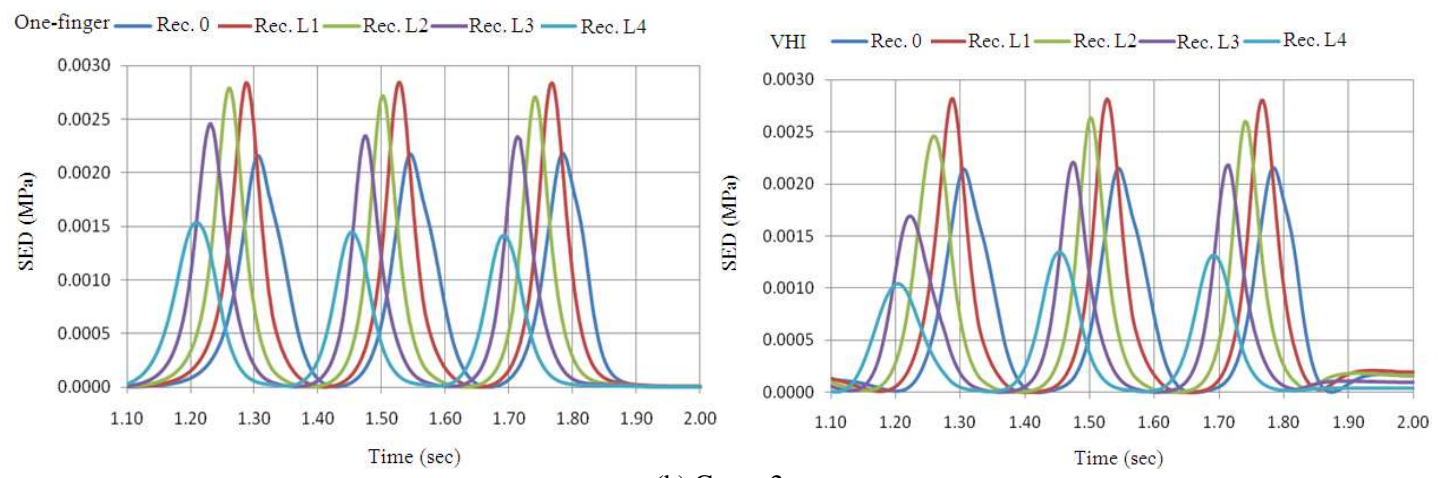

(b) Group 2

Fig. 4: SED at location of SAI receptors on left side of fingertip in addition to center receptor (Fig. 2) VHI case (right) and one-finger case (left); (a) Recs. L5 L8, (b) Recs. L1 L4 and Rec. 0 

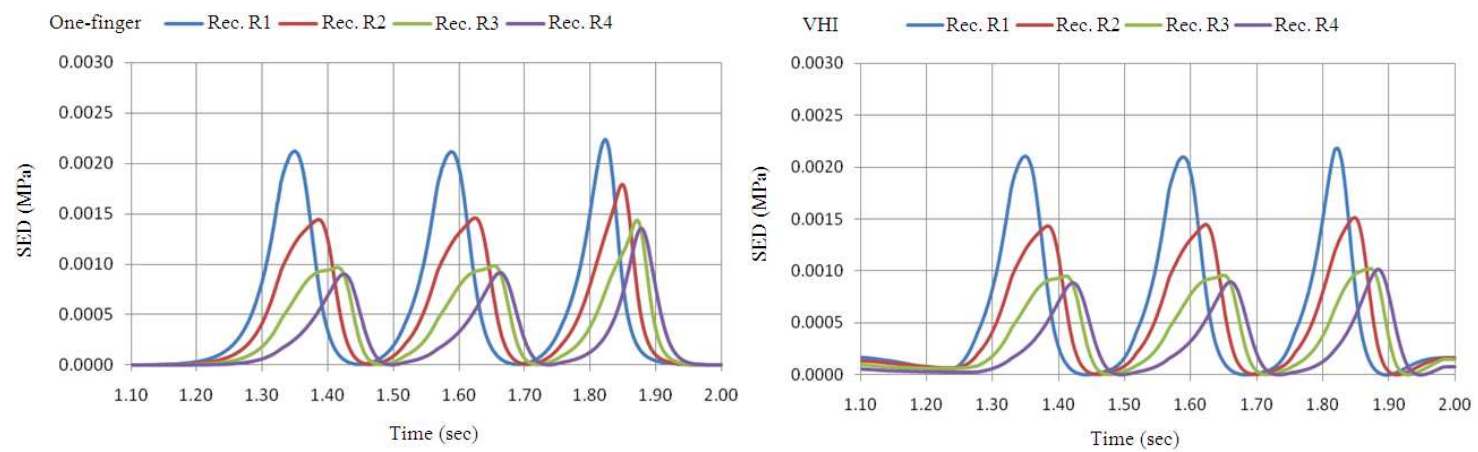

(a) Group 1
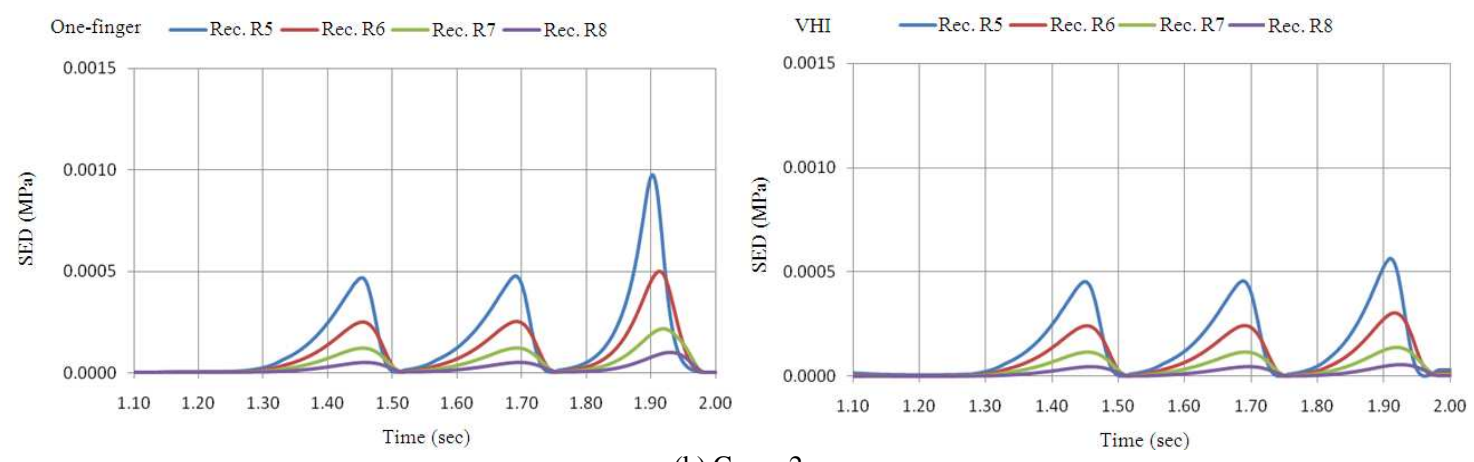

(b) Group 2

Fig. 5: SED at the location of SAI receptors on right side of fingertip (Fig. 2) for VHI case (right) and one-finger case (left); (a) Recs. R1 R4, (b) Recs. R5 R8

In Fig. 5a, the responses obtained from Recs. R3 and R4 to the last wire for the VHI case are about $25 \%$ less than those for the one-finger case. In Fig. 5b, when the last wire passes, the responses obtained from Recs. R5 and R6 for the VHI case are almost half of those for the one-finger case.

We believe that the decrease of the response of the SAI receptors illustrated above plays a role in the perception of VHI mainly due to the decrease of the feeling of the wires touched. The main difference of the response between the two cases is for both the first wire when it starts to touch the fingertip and the last wire when it leaves the fingertip.

In this study we only modeled one finger where the actual illusion happens on all the fingers of both hands and the actual illusion happens when rubbing but we only modeled passing wires over the fingertip. The importance of the first and last wires becomes clear, because in the actual illusion there is always a wire that is starting to touch or going to leave one of the fingers.

The change from the touch to no touch state is also important because we believe that the response of the Fast Adapting type I (namely FAI) receptors that fire only when the touch state changes could be related to the perception of VHI.

\section{CONCLUSION}

Even with a simple model we illustrated how the response of SAI afferents could play a role in the perception of VHI. The response of some SAI receptors is reduced by up to $50 \%$, which means that the perception of the wires is reduced compared with the case of simply touching them. This study could be used to design a haptic display that uses the VHI mechanism.

\section{ACKNOWLEDGEMENT}

The researchers thank Professor Tetsu Miyaoka for his insightful discussions that made this study possible.

\section{REFERENCES}

Bresciani, J., K. Drewing and M. Ernst, 2008. Human Haptic Perception and the Design of HapticEnhanced Virtual Environments. In: The Sense of Touch and its Rendering, Bicchi, A., M. Buss, M.O. Ernst and A. Peer (Eds.). Springer-Verlag Berlin, Heidelberg, ISBN: 978-3540790341, pp: 61-106. 
Dandekar, K., B.I. Raju and M.A. Srinivasan, 2003. 3-d finite-element models of human and monkey fingertips to investigate the mechanics of tactile sense. J. Biomech. Eng., 125: 682-691.

Gerling, G.J. and G.W. Thomas, 2005. The effect of fingertip microstructures on tactile edge perception. Proceeding of the 1st Joint Eurohaptics Conference and Symposium on Haptic Interfaces for Virtual Environment and Teleoperator Systems, Mar. 18-20, IEEE Computer Society, Washington DC, USA, pp: 63-72. DOI: 10.1109/WHC.2005.129

Gerling, G.J. and G.W. Thomas, 2008. Fingerprint lines may not directly affect SA-I mechanoreceptor response. Somatosensory Motor Res., 25: 61-76. http://www.informaworld.com/index/791486612.pdf

Hayward, V., 2008. A brief taxonomy of tactile illusions and demonstrations that can be done in a hardware store. Brain Res. Bull., 75: 742-752. DOI: 10.1016/j.brainresbull.2008.01.008

Herrera, R.M., 2007. A bio-inspired method for incipient slip detection. Lecture Notes Comput. Sci., 4830: 347-356. DOI: 10.1007/978-3-54076928-6_36

Johansson, R.S. and J.R. Flanagan, 2009. Coding and use of tactile signals from the fingertips in object manipulation tasks. Nat. Rev. Neurosci., 10: 345-359.

http://www.nature.com/nrn/journal/vaop/ncurrent/f ull/nrn2621.html

Lesniak, D.R. and G.J. Gerling, 2009. Predicting SA-I mechanoreceptor spike times with a skin-neuron model. Math. Biosci., 220: 15-23. DOI: 10.1016/j.mbs.2009.03.007

Maeno, T., K. Kobayashi and N. Yamazaki, 1998. Relationship between the structure of human finger tissue and the location of tactile receptors. JSME Int. J. Ser. C-Dyn. Control Robot. Des. Manuf., 41: 94-100. http://ci.nii.ac.jp/naid/110004155855
Mochiyama H., A. Sano, N. Takesue, R. Kikuuwe and K. Fujita et al., 2005. Haptic illusions induced by moving line stimuli. Proceeding of the 1st Joint Eurohaptics Conference and Symposium on Haptic Interfaces for Virtual Environment and Teleoperator Systems, Mar. 18-20, IEEE Computer Society, Washington DC, USA, pp: 645-648. DOI: 10.1109/WHC.2005.129

Phillips, J.R. and K.O. Johnson, 1981. Tactile spatial resolution. II. Neural representation of bars, edges and gratings in monkey primary afferents. J. Neurophysiol., 46: 1192-1203. PMID: 6275041

Scilingo, E.P., N. Sgambelluri and A. Bicchi, 2008. The Role of Tactile Flow in Processing Dynamic Haptic Stimuli. In: The Sense of Touch and its Rendering, Bicchi, A., M. Buss, M.O. Ernst and A. Peer (Eds.). Springer-Verlag, Berlin Heidelberg, ISBN: 9783540790341, pp: 39-60.

Srinivasan, M.A., 1989. Surface deflection of primate fingertip under line load. J. Biomech., 22: 343-349. PMID: 2745468

Sripati, A.P., S.J. Bensmaia and K.O. Johnson, 2006. A continuum mechanical model of mechanoreceptive afferent responses to indented spatial patterns. J. Neurophysiol., 95: 3852-3864. PMCID: PMC1839063/

Wu, J.Z., K. Krajnak, D.E. Welcome and R.G. Dong, 2006. Analysis of the dynamic strains in a fingertip exposed to vibrations: Correlation to the mechanical stimuli on mechanoreceptors. J. Biomech., 39: 2445-2456. DOI: 10.1016/j.jbiomech.2005.07.027

Zhang, Y., Y. Mukaibo and T. Maeno, 2006. A multipurpose tactile sensor inspired by human finger for texture and tissue stiffness detection. Proceeding of the IEEE International Conference on Robotics and Biomimetics, Dec. 17-20, IEEE Computer Society, Washington DC, USA, pp: 159-164. DOI: 10.1109/ROBIO.2006.340351 